\title{
Primer registro para el Perú de Nematophila grandis (Diesing, 1839) Travassos, 1934 (Trematoda, Diplodiscidae) en Podocnemis unifilis (Troschel, 1848) (Testudines, Pelomedusidae)
}

\author{
First record for Peru of Nematophila grandis (Diesing, 1839) Travassos, 1934 \\ (Trematoda, Diplodiscidae) in Podocnemis unifilis (Troschel, 1848) \\ (Testudines, Pelomedusidae)
}

\author{
Patricia Salízar y Lidia Sánchez*
}

Presentado: $02 / 06 / 2004$

Aceptado: $\quad 15 / 07 / 2004$

\section{Resumen}

El presente trabajo registra por primera vez para el Perú a Nematophila grandis (Diesing, 1839) Travassos, 1934, en la tortuga de río Podocnemis unifilis «taricaya». Los hospederos fueron colectados en las localidades del Río Putumayo, Samiria, Iquitos (Loreto) y Manu (Madre de Dios). El material identificado pertenece a la Colección Helmintológica del Departamento de Protozoología, Helmintología e Invertebrados Afines del Museo de Historia Natural de la Universidad Nacional Mayor de San Marcos.

Palabras Claves: Nematophila grandis, Diplodiscidae, Podocnemis unifilis, Loreto, Madre de Dios.

\section{Abstract}

The present work registers for the first time for the Peru to Nematophila grandis (Diesing, 1839) Travassos, 1934 in the Yellow-spotted River Turtle Podocnemis unifilis «taricaya». The hosts were collected from the localities of the River Putumayo, Samiria, Iquitos (Loreto) and Manu (Madre de Dios). Identified material was deposited in the Helmintological Collection of the Department of Protozoology, Helmintology and Relationed Invertebrates of the Natural History Museum of San Marcos University.

Keywords: Nematophila grandis, Diplodiscidae, Podocnemis unifilis, Loreto, Madre de Dios

\section{Introduccion}

Podocnemis unifilis (Diesing, 1839) Travassos, 1934, se distribuye a todo lo largo de nuestra Amazonía, y su fauna helmintológica es poco conocida. Especies de Nematophila han sido reportadas para diferentes tortugas dulceacuícolas de Centro y Sudamérica. Travassos (1934) reporta Nematophila grande en tortugas del valle del Amazonas incluyendo Chelys fimbriata; Hydraspis geoffroyana; $H$. gibba; $H$. schopjii; Podocnemis dumeriliana; $P$. expansa; P. tracaxa; Rhynemis nasuta. Posteriormente, Lent y Teixeira de Freitas (1939)

*Museo de Historia Natural Universidad Nacional Mayor de San Marcos. Av. Arenales 1256, Jesús María-Apartado 14-0434. Lima-Perú.

E-mail Patrcia Salízar: vasquezpt@yahoo.com.mx reportaron a $N$. grande en el intestino delgado y grueso de $P$. expansa en el Estado de Pará (Brasil). Caballero, Zerecero y Grocott (1958) describen a $N$. grande en el intestino delgado de Rhinoclemmys melanosterna para la ciudad de Panamá. Yamaguti (1971) refiere el trabajo de Cordero y Vogelsang (1940) donde registran a Nematophila venezuelensis (=Allassostoma venezuelensis) y $N$. ovalis en Podocnemis sp. para Venezuela, así mismo incluye el trabajo de Alho (1964) quien reporta a $N$. grande en el intestino delgado de Kinosternon scorpioides scorpioides y Geoemyda punctularia punctularia en Brasil. Por último, Dyer y Carr (1990) reportan a N. grandis para Rhinoclemmys areolata y R. nasuta en Ecuador; R. punctularia de Guyana Francesa y $R$. areolata de México. 


\section{Material y métodos}

Los hospederos fueron colectados en los años 1960 en Iquitos, 1968 en Río Putumayo, 1977 en la provincia de Manu y 1980 en Río Samiria. Los tremátodos fueron colectados por la Dra. Luz Sarmiento, fijados en alcohol de $70^{\circ}$ y depositados en la Colección Helmintológica del Departamento de Protozoología, Helmintología e Invertebrados Afines del Museo de Historia Natural. Para su identificación algunos especímenes fueron coloreados con Carmín de Semichron, deshidratados y aclarados en Creosota. Luego fueron montados en Permount. Las medidas fueron realizadas con

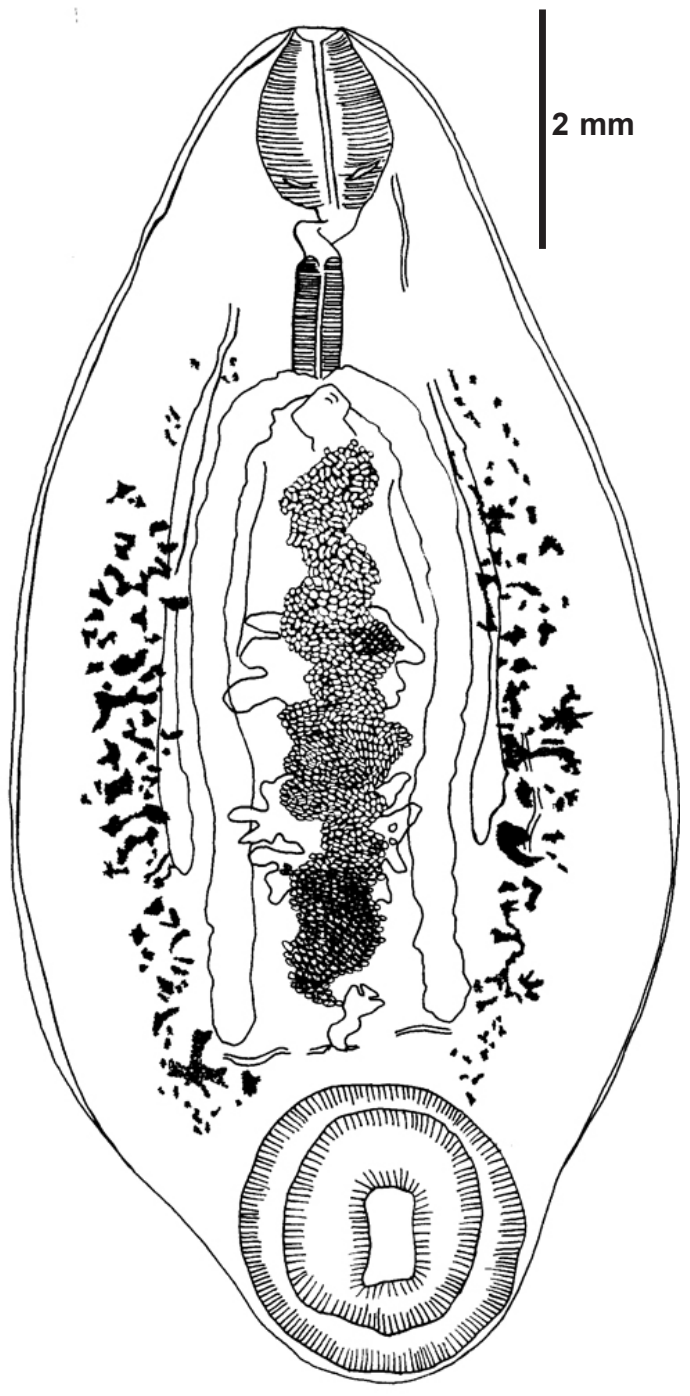

Figura 1. Nematophila grandis ayuda del ocular micrométrico y expresadas en milímetros, se consideraron el diámetro de las ventosas y longitud y ancho del cuerpo, tanto de los especímenes montados como los preservados en alcohol. El resto de medidas como la longitud de la pre-faringe y faringe, se realizaron sólo en los especímenes montados. Los dibujos fueron hechos con ayuda del microscopio invertido.

La identificación de los parásitos se hizo con ayuda de las Claves Taxonómicas de Travassos, Freitas y Kohn (1969) y Thatcher (1993).

\section{Resultados}

Se estudiaron 18 especímenes del tremátodo Nematophila grandis localizados en el estómago e intestino de Podocnemis unifilis provenientes de Loreto y Madre de Dios.

Este trabajo constituye un nuevo registro para diferentes localidades del Perú. Además se da a conocer a Podocnemis unifilis como nuevo hospedador.

\section{Taxonomía y Descripción}

\begin{tabular}{|c|c|}
\hline ClaAse & TREMATODA RudOLPHI, 1808. \\
\hline ORDEN & $\begin{array}{l}\text { PARAMPHISTOMIFORMES TRAVASSOS, } \\
\text { FREITAS Y KOHN, } 1969 .\end{array}$ \\
\hline & DiplodiscidAe SkRJABIN, 1949. \\
\hline IILIA & NEMATOPHILINAE SkRJABIN, 1949. \\
\hline
\end{tabular}

Amphistoma grande Diesing, 1839

Amphistomun grande Diesing, 1851

Amphistoma grande Leidy, 1988

Nematophila grande Travassos, 1934

Nematophila grandis Yamaguti, 1971

\section{Nematophila grandis (Diesing, 1839) Travassos, 1934 \\ (Fig.1)}

La longitud del cuerpo varía de 5,5 a 29 $\mathrm{mm}$ y el ancho de 2 a $14 \mathrm{~mm}$. Cutícula lisa. Ventosa oral terminal 0,5 a 2,5 $\mathrm{mm}$ x 0,5 a 1,5 $\mathrm{mm}$, posee divertículos rudimentarios en la espesura de sus paredes. Acetábulo terminal con 0,75 a $3 \mathrm{~mm}$ x 0,75 a 3,5 mm. Pre-faringe con 0,25 a $2 \mathrm{~mm}$ de longitud, unido a una faringe cilíndrica, muscular con 0,5 a 3,5 mm de 
longitud y 0,5 a $1,5 \mathrm{~mm}$ de ancho. Esófago muy corto (Fig. 2). Asas intestinales bifurcadas, poco sinuosas que terminan a la altura de la zona ovárica. Bolsa del cirro rudimentario. Espermiducto sinuoso extendiéndose desde la bolsa del cirro hasta el testículo posterior. Testículos pareados levemente separados, uno posterior al otro, ramificados, pre-ováricos localizados entre las asas intestinales. Un único ovario post-testicular entre las asas intestinales. Útero entre asas intestinales, se extiende de la zona ovárica hasta la bolsa del cirro. Vitelógenas constituídas por pequeños y numerosos folículos externos a las asas intestinales, que se extienden de la zona de la faringe a la zona acetabular; dos pequeños viteloductos transversales que salen de la porción final de las glándulas vitelógenas hacia el extremo posterior del ovario. Sistema linfático constituído por dos pares de troncos longitudinales paralelas a las asas intestinales, externos e internos.

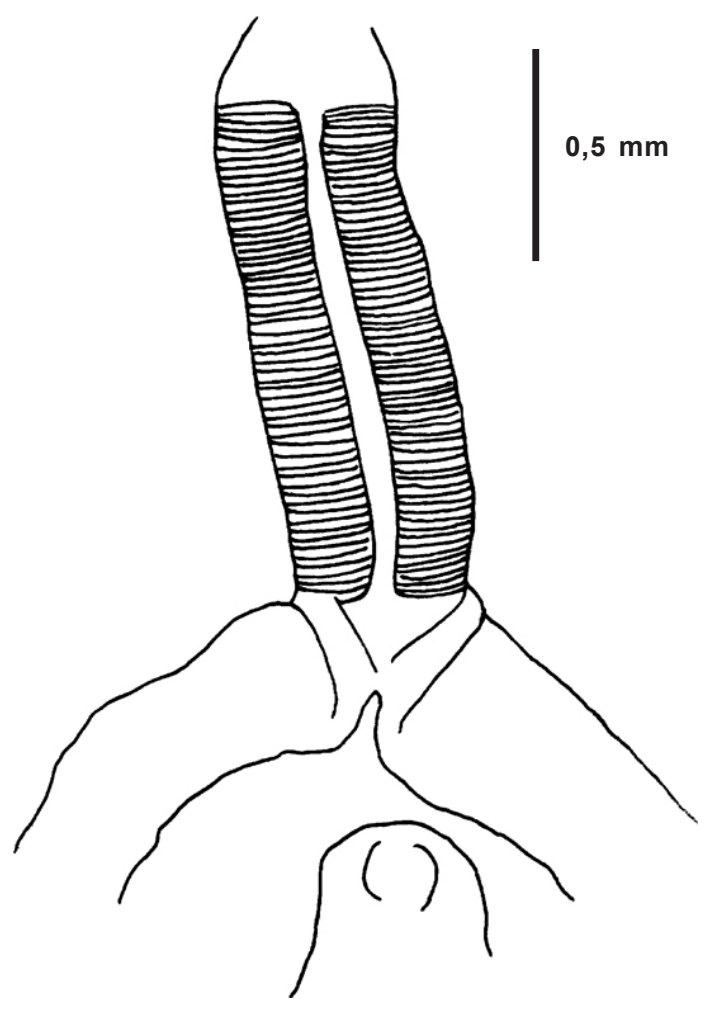

Figura 2. Faringe muscular, esófago corto y cirro rudimentario de Nematophila grandis

\section{Comentarios}

Las características diferenciales entre los tremátodos reportados para Podocnemis expansa son como siguen: en Podocnemitrema papillosus (Alho y Vicente, 1964) se observa la ausencia de acetábulo y faringe pequeña, en cambio $N$. grandis presenta acetábulo fuerte y una faringe muscular y cilíndrica. En Halltrema avitellina (Lent y Freitas, 1939) se distingue cutícula con pequeñas proyecciones papiliformes, ventosa oral con divertículos grandes, faringe ausente, esófago estrecho, ventosa genital presente; a diferencia de $N$. grandis, que presenta cutícula lisa, ventosa oral con divertículos rudimentarios, esófago corto y ventosa genital ausente.

Cordero y Vogelsang describieron en 1940 nuevas especies de paramfistómidos de Podocnemis sp. de Venezuela: Allassostoma venezuelensis y Nematophila ovalis; el examen y estudio realizado por Caballero, Zerecero y Grocott en 1958 concluyen que estas especies son sinónimas de Nematophila grande por coincidir con características de esta última.

Por las características expuestas anteriormente, concluimos que se trata de Nematophila grandis, la cual amplia su distribución a diversas localidades de la amazonía peruana y se presenta a Podocnemis unifilis como nuevo hospedador.

\section{Agradecimientos}

Queremos expresar nuestro agradecimiento al Biólogo César Aguilar Puntriano del Departamento de Herpetología del Museo de Historia Natural UNMSM por su orientación en la clasificación taxonómica del hospedero. A Patricia Pilitt, Curador Asociado de la Colección Nacional de Parásitos de USA, por facilitarnos referencias bibliográficas. Al Biólogo Leonardo Romero Profesor Asociado de la Facultad de Biología de UNMSM por su gentileza en la toma de las fotografías de los especímenes. 


\section{Literatura citada}

Caballero E.; C. Zerecero, y R. Grocott. 1958. Helmintos de la Republica de Panamá. XXI. Algunos Tremátodos de Quelonios de Agua Dulce $2^{\mathrm{a}}$ parte. Ann. Inst. Biol. Mex. XXIX:181-202.

Dyer W. y J. Carr. 1990. Some Digeneas of the Neotropical Turtle Genus Rhinoclemmys in Mexico and South America. J. Helminthol. Soc. Wash. 57(1):12-14.

Lent H. y J. F. Teixeira De Freitas. 1939. Pesquisas Helmintológicas Realizadas no Estado do Pará. VII. Trematoda. Paramphistompidea. Boletim Biológico 4(1):82-86.
Thatcher V. 1993. Trematódeos Neotropicais. Inst. Nac. Pesq. Amaz. Manaus. 553 p.

Travassos L. 1934. Synopse dos Paramphistomoidea. Memorias do Instituto Oswaldo Cruz. 29(1):85-87.

Travassos L.; L. F. Teixeira De Freitas y A. Kohn 1969. Trematódeos do Brasil. Mem. Inst. Oswaldo Cruz. 67(1):623-264.

Yamaguti S. 1971. Synopsis of Digenetic Trematodes of Vertebrates. Vol. I. Reigabu Publishing Co. Tokio. 1074 p. 